\title{
The perivascular space of the central retinal artery as a potential major cerebrospinal fluid inflow route: implications for optic disc edema in astronauts
}

\author{
Peter Wostyn ${ }^{1} \cdot$ Thomas H. Mader ${ }^{2} \cdot$ Charles Robert Gibson $^{3} \cdot$ Hanspeter Esriel Killer ${ }^{4,5}$
}

Received: 12 August 2019 / Accepted: 3 September 2019 / Published online: 20 September 2019

(c) The Royal College of Ophthalmologists 2019

\section{To the Editor:}

In a recent paper published in Eye [1], we hypothesized that optic disc edema in astronauts may result, at least partly, from the forcing of perioptic cerebrospinal fluid (CSF) into the optic nerve (ON) and optic disc along perivascular spaces surrounding the central retinal artery (CRA). For this hypothesis to be true, it is essential that the subarachnoid space (SAS) of the ON communicates with the perivascular spaces of the CRA. Whether or not such communication exists, is still a matter of debate with some studies denying the presence of any continuity [2], while others suggest the existence of a connection [3]. Now, a new study by Jacobsen et al. [4] provides evidence for a direct communication between CSF in the SAS and the extravascular space of the human visual pathway, suggesting a paravascular inflow route.

Jacobsen et al. [4] performed an MRI study of human visual pathway structures following intrathecal administration of gadobutrol. CSF tracer enrichment was found within the ON, optic chiasm, optic tract, and primary visual cortex. Although the authors could not rule out diffusion of gadobutrol from CSF, they argued that a plausible explanation for their observations was that gadobutrol, equivalent to the

\footnotetext{
Peter Wostyn

wostyn.peter@skynet.be

Department of Psychiatry, PC Sint-Amandus, Beernem, Belgium

2 Colonel, US Army (retired), Moab, UT, USA

3 Coastal Eye Associates, Webster, TX, USA

4 Department of Ophthalmology, Cantonal Hospital Aarau, Aarau, Switzerland

5 Department of Biomedicine, University of Basel, Basel, Switzerland
}

rodent studies by Mathieu et al. [5], entered the optic pathway through glymphatic paravascular spaces. Interestingly, substantial gadobutrol enrichment was seen in the retrobulbar part of the $\mathrm{ON}$, as compared with the middle and posterior sections, corresponding to the entrance of the CRA. As noted by the authors, analogous to the main entry routes of CSF tracer molecules into the human brain parenchyma along large artery trunks at the brain surface, this area could function as a major periarterial route facilitating the entry of gadobutrol from the SAS into the ON interstitium. We believe that such glymphatic perivascular entry of CSF along the CRA may be even more relevant under conditions of prolonged microgravity, where CSF outflow routes (venous and lymphatic) from the SAS of the ON may be impacted by headward fluid shifts. Therefore, the findings of the study by Jacobsen et al. [4] may open new perspectives for research into a possible CSF-related glymphatic mechanism underlying microgravity-induced optic disc swelling.

\section{Compliance with ethical standards}

Conflict of interest The authors declare that they have no conflict of interest.

Publisher's note Springer Nature remains neutral with regard to jurisdictional claims in published maps and institutional affiliations.

\section{References}

1. Wostyn P, Mader TH, Gibson CR, Killer HE. The escape of retrobulbar cerebrospinal fluid in the astronaut's eye: mission impossible? Eye. 2019. https://doi.org/10.1038/s41433-019-0453-8.

2. Hayreh SS. The sheath of the optic nerve. Ophthalmologica. 1984;189:54-63.

3. Wostyn P, Killer HE, De Deyn PP. Glymphatic stasis at the site of the lamina cribrosa as a potential mechanism underlying openangle glaucoma. Clin Exp Ophthalmol. 2017;45:539-47. 
4. Jacobsen HH, Ringstad G, Jørstad $\varnothing \mathrm{K}$, Moe MC, Sandell T, Eide PK. The human visual pathway communicates directly with the subarachnoid space. Invest Ophthalmol Vis Sci. 2019; 60:2773-80.
5. Mathieu E, Gupta N, Ahari A, Zhou X, Hanna J, Yücel YH. Evidence for cerebrospinal fluid entry into the optic nerve via a glymphatic pathway. Invest Ophthalmol Vis Sci. 2017;58: 4784-91. 